\title{
The Effects of Combined Exercise Program on the Body Composition, Metabolic Syndrome Risk Factor in Middle-aged Women
}

\author{
Ryewon Ma1)
}

\begin{abstract}
The purpose of this study was to investigate the effects of combined exercise on the health self-efficacy, exercise adherence, body composition, and blood lipid in middle-aged women and to provide fundamental data to the development and application of combined exercise program for continuous health management. The one group pre-post experimental design was applied and the subjects were 20 middle-aged women aged 35 to 65 who participated in a combined exercise program administered by the $\mathrm{S}$ District Public Health Center in Busan Metropolitan City. The proposed program was a 12-weeks exercise program composed of aerobic exercise, low-middle intensity muscle-strength exercise, and flexibility and balance exercise. The effectiveness of the program was measured by using structured questionnaire on health self-efficacy and exercise adherence. The body composition ere measured using height, weight, waist circumference, skeletal muscle mass, and body fat mass, and in addition, blood lipid test measured cholesterol, triglyceride, and blood glucose. The collected data were analyzed by using SPSS 23.0 program and the descriptive statistics and Wilcoxon sign rank were conducted. The proposed combined exercise program significantly increased the nutrition efficacy, a sub-scale of health self-efficacy and exercise habit and exercise concern, the sub-scales of willingness of exercise adherence of subjects. For the body composition, the waist circumference and body fat mass decreased significantly and the skeletal muscle mass increased. The results of blood lipid test showed significant decrease in triglyceride and cholesterol. The combined exercise program in middle-aged women decreased the level of triglyceride and cholesterol and waist circumference, which are risk factors of metabolic syndrome, and had positive effects on health self-efficacy and exercise adherence. The proposed program is expected to raise the willingness to participate in health management in middle-aged women who are experiencing many physical and psychological changes by providing positive effect on their health management and inducing continuous exercise adherence.
\end{abstract}

Keywords: Middle-aged Women, Combined Exercise, Body Composition, Health Self-Efficacy, Exercise Adherence

\section{Introduction}

The development of medicine and science technology in modern society has prolonged the Received(October 17, 2019), Review Result(1st: November 07, 2019, 2nd: December 19, 2019), Accepted(February 20, 2020)

1) (Professor) 51767 Dept. Nursing, Kyungnam Univ, Kyungnamdaehak-ro 7, Masanhappo-gu, Changwon-si, Gyeongsangnam-do, Republic of Korea

email: mrw4023@kyungnam.ac.kr 
The Effects of Combined Exercise Program on the Body Composition, Metabolic Syndrome Risk Factor in Middleaged Women

life span of human beings and ensured the convenience in life and abundant life; however, such convenience caused decrease in physical activity and thus population with obesity and metabolic syndrome has rapidly increased[1][2].

Women have lower basal metabolism and energy expenditure, accumulate fat more easily with aging, and after menopause and decrease in female hormones, resulting in less muscle and increase in fatty tissue[3]. In middle-aged women, in particular, the risk of metabolic syndrome increases rapidly due to reduced fat mass and physiological aging[4] and increased body fat mass in the upper body[5]. It is known that the metabolic syndrome, a cause of simultaneous disorders such as abdominal obesity, hypertension, insulin resistance, and hyperlipemia, increases the risk of diabetes, cardiovascular disease, and mortality[1][6]. The middle-aged women, being at a point between maturity and old age in terms of life stages, may be exposed to various diseases unless they are not directly managed[7], and, in this period, the importance of health management is emphasized because it affects quality of life in old age.

The exercise, a regular physical activity, improves physical health[8] and decreases the level of depression and stress by decreasing body fat mass, increasing muscle mass, and maintaining basic metabolism[9]. The exercise, especially in middle-aged women, is an important factor in improving the quality of life by preventing menopausal symptoms and chronic diseases, positively affecting physical fitness and body composition, and improving self-esteem[5][10-12]. The complex exercise is a combination of two or more types of exercise. It is a movement that has characteristics of both aerobic and anaerobic exercises by performing both aerobic exercise and resistance exercise using dumbbell[13]. The aerobic exercise is effective in preventing coronary artery disease by improving the respiratory system and blood lipid metabolism and the muscle resistance exercise has effect of increasing the basal metabolism, preventing bone loss, and increasing bone mineral density[14]. It is recommended, therefore, to perform the aerobic, anaerobic, and resistance exercises together rather than individually[15]. The effect of combined exercise has been studied extensively based on the results that it has a stronger effect of reducing metabolic syndrome factors, improving cardiovascular fitness, and reducing cardiovascular risk factors[16-18].

The exercise of middle-aged women has a close relationship with lifestyle[10]. Although it is important to recognize the importance of exercise effects, it is not easy to practice exercises continuously, so exercise is often stopped before exercising. In fact, $60 \%$ of middle-aged women are not able to participate in the exercise[19], so it is very important 
for them to choose the exercise they need and to actively participate in the exercise. Health self-efficacy is an important variable in sustaining exercise behaviors as the individual's perceived level of ability related to the physical performance for health promotion[19-22]. Although the importance of exercise is emphasized as a method for health management of middle-aged women, there are very few studies to confirm the development and effect of exercise programs that actively participate in and continue to participate in these exercise programs.

The purpose of this study was, therefore, to develop a combined exercise program to induce the participation of middle-aged women and to examine the effect of the participation in the proposed program on the predictive factors such as body composition and blood lipid, and health self-efficacy and exercise adherence. This study also intended to provide fundamental data on the development of effective exercise programs for the continuous health care of middle-aged women.

\section{Method}

\subsection{Subjects}

The subjects of this study were 20 middle-aged women aged 35 to 65 who participated in a combined exercise program administered by the $S$ District Public Health Center in Busan Metropolitan City. The contents, procedures, and associated risks of the exercises and the principles of voluntary participation and free abandonment were explained in detail before acquiring informed consent and enrolling the study. The data were collected for about three months from 4th Sep. to 29th Nov., 2017.

\subsection{Procedure}

This one group pre-post experimental study intended to investigate the effects of 12-weeks combine exercise program on body composition, blood lipid, health self-efficacy, and exercise adherence in middle-aged women. The exercise program was developed based on the study of Cholewa et al.[20] applying the same strength training method, number of times, and number of sets according to the guidelines of NSCA(2008). The general characteristics, body composition, blood lipid, health self-efficacy, and exercise adherence were pre-measured one week before the exercise program and post-measurement was performed over the last week of 
The Effects of Combined Exercise Program on the Body Composition, Metabolic Syndrome Risk Factor in Middleaged Women

the exercise program. To minimize the effects of extraneous variables on the results of this study, telephone counseling was added during the program to instruct them to keep their eating habits unchanged during program period and encouraged them not to participate in additional exercise.

\subsection{Measurement}

\subsubsection{Body Composition}

The height and weight of subjects were measured using automatic body measure(Fanics, FE810). The waist circumference was measuring on middle point between lower border of rib cage and top of iliac crest at end of normal expiration using tape measure(SECA200, SECA, Germany). The body fat percentage(\%fat) and skeletal muscle mass, as a body composition ratio, were measured using the Inbody 4.0 Body Composition Analyzer.

\subsubsection{Blood Lipids Test}

The blood samples of subjects were collected at 9 am using a single-use syringe in antecubital vein after nine hours of fasting. HDL, LDL, TG and T-cholesterol were analyzed by enzymatic colorimetric assay using the Modular analytics PE(Poche, Germany). The glucose-related variables were analyzed by UV Assay(HK) method using Modular analytics PE(Poche, Germany).

\subsubsection{Health Self-Efficacy}

The health self-efficacy was measured using 28 items from the Self-Rated Abilities for Health Practices Scale[21] revised and translated by Choi[22]. The higher score indicates higher degree of heal self-efficacy.

\subsubsection{Health Self-Efficacy Exercise Adherences}

The Willingness to Adhere Exercise-Korean version developed by Choi[23] was used. The tool consists of 17 items and measures five sub-scales of exercise ability, exercise habits, exercise environment, exercise interest, and exercise friend. The higher score indicates the higher level of direct participation or adherence without stopping.

\subsection{Combined Exercise Intervention}

A total of 20 middle-aged women attending to $S$ District Public Health Center in Busan 
Metropolitan City were face-to-face interviewed to develop proposed program and, based on them, four specialists including a doctor specialized in physical education, an exercise prescription specialist, one social work practitioner, and a doctor specialized in adult nursing developed the detail contents of program. The program was, reflecting the strength of combined exercise, composed of aerobic exercise, low-middle intensity exercise, and balance exercise. The stretching as a warm-up and cool-down exercises before and after main exercise was added. Warm-up and cool-down sessions were performed for 10-20 seconds of dynamic and static stretching. The main exercises include aerobic exercise (body exercise, stretching, step box), low-middle intensity exercise (core exercise, thera-band, kettle-bell), and flexibility and balance exercise (yoga). The program consisted of three sessions per week for 12 weeks and it takes about 60 minutes in completing a session. The total exercise time was 60 minutes, and 10 minutes of warm-up and cool-down exercises were performed before and after the main exercise. Low-middle intensity exercise was performed at 50-60\% 1RM, 2-3 sets (13-15 times per set). The exercise program was conducted three times a week. And combined exercise including 1 day lower body strength / flexibility exercise (squats, leg extensions, step-ups/box, hip thrust, modified yoga), '2 day' upper body strength / flexibility exercise (thera-band, seated cable row, cable press down, kettle-bell, modified yoga), '3 day' lower-upper body exercise and whole-body exercise (thera-band, lat pull-down, leg press, leg curls, modified yoga) were performed. After the first set, they were allowed to rest for a minute or two to get enough breath back.

To satisfy the weight control demand of subjects, the diet information was provided and the health education was provided once a week in group format to promote motivation and interests of subjects. The various health information, in addition, were provided through mobile apps.

\subsection{Statistic}

The collected data were analyzed using SPSS 23.0 program. The pre-and post-program mean and standard deviations of the subjects' health self-efficacy and exercise adherence were used in the level of the corresponding variables. The Wilcoxon signed-rank test was conducted to test the test the ranks of pre-and post-experimental effect.

\section{Result}


The Effects of Combined Exercise Program on the Body Composition, Metabolic Syndrome Risk Factor in Middleaged Women

\subsection{Subjects}

The mean age of the subjects was $58.35( \pm 7.17)$ years old. The educational levels were under elementary school $5 \%(\mathrm{n}=1)$, middle school graduation $25 \%(\mathrm{n}=5)$, high school graduation $45 \%(n=9)$, and university graduation and over $25 \%(n=5)$. The marital status were unmarried $5 \%(n=1)$, married $90 \%(n=18)$, and divorced $5 \%(n=1)$.

\subsection{Difference in Body Composition and Blood Test between before and after Program}

[Table 1] shows the body composition and blood test results of subjects before and after exercise program. The waist circumference decreased significantly from $83.18 \pm 6.70 \mathrm{~cm}$ before program to $81.15 \pm 6.59 \mathrm{~cm}$ by $2.03 \pm 0.1 \mathrm{~cm} \quad(\mathrm{Z}=-3.47, \mathrm{p}<.001)$. The skeletal muscle mass significantly increased after program by $.42 \pm .15 \mathrm{~kg}(\mathrm{Z}=-2.52, \mathrm{p}<.01)$ and the body fat decreased significantly after program by $.90 \pm .04 \mathrm{~kg}(\mathrm{Z}=-2.22, \mathrm{p}<.01)$. The results of blood lipid test showed that triglyceride (TG) significantly decreased after program by $18.50 \pm 1.07 \mathrm{mg} / \mathrm{dl} \quad(Z=-2.91$, $\mathrm{p}<.001)$. The total cholesterol and LDL cholesterol decreased significantly by $16.00 \pm .90 \mathrm{mg} / \mathrm{dl}$ $(\mathrm{Z}=-3.47, \mathrm{p}<.001)$ and $12.57 \pm 3.05 \mathrm{mg} / \mathrm{dl}(\mathrm{Z}=-3.47, \mathrm{p}<.001)$, respectively.

[Table 1] Effectiveness of Exercise on Body Composition and Blood Lipids Test

\begin{tabular}{|c|c|c|c|c|c|c|c|}
\hline & \multirow{2}{*}{$\begin{array}{c}\text { Variable } \\
\text { Weight(kg) }\end{array}$} & \multicolumn{2}{|c|}{$\begin{array}{c}\text { Pre } \\
\mathrm{M}(\mathrm{SD})\end{array}$} & \multicolumn{2}{|c|}{$\begin{array}{c}\text { Post } \\
\text { M(SD) }\end{array}$} & \multirow{2}{*}{$\begin{array}{c}\mathrm{Z} \\
-0.50\end{array}$} & \multirow{2}{*}{$\begin{array}{c}\mathrm{P} \\
0.614\end{array}$} \\
\hline \multirow{5}{*}{$\begin{array}{c}\text { Body } \\
\text { Composition }\end{array}$} & & 62.56 & 9.50 & 62.13 & 9.22 & & \\
\hline & WaistCircumferenceLine $(\mathrm{cm})$ & 83.18 & 6.70 & 81.15 & 6.59 & $-3.47^{* * *}$ & 0.001 \\
\hline & Body Mass Index & 24.26 & 2.95 & 24.11 & 2.84 & -0.69 & 0.491 \\
\hline & Skeletal Muscle Mass(kg) & 23.11 & 3.97 & 23.53 & 3.82 & $-2.52^{* *}$ & 0.012 \\
\hline & Body Fat(kg) & 20.05 & 5.20 & 19.15 & 5.16 & $-2.22^{* *}$ & 0.027 \\
\hline \multirow{5}{*}{ Blood Lipids } & Glucose(mg/dl) & 112.60 & 21.58 & 108.30 & 20.94 & -1.17 & 0.242 \\
\hline & HDL-Cholesterol(mg/dl) & 58.15 & 15.76 & 59.10 & 15.29 & -0.71 & 0.480 \\
\hline & Triglycerides(mg/dl) & 121.50 & 56.86 & 103.00 & 55.79 & $-2.91^{* * *}$ & 0.004 \\
\hline & Total-Cholesterol(mg/dl) & 206.40 & 48.34 & 190.40 & 49.24 & $-2.17^{*}$ & 0.030 \\
\hline & LDL-Cholesterol(mg/dl) & 141.35 & 39.98 & 128.70 & 43.03 & $-2.03^{*}$ & 0.042 \\
\hline
\end{tabular}

3.3 Difference in Health Self-Efficacy and Exercise Adherence between before and after Program

The nutritional efficacy from the health self-efficacy significantly increased after program 
$(Z=-2.25, p<.05)$. The exercise habit $(Z=-2.63, p<.01)$, exercise concern $(Z=-3.06, p<.01)$ from the exercise adherence showed significant difference before and after program the willingness to exercise adherence significantly increased $(Z=-2.75, p<.01)$ [Table 2].

[Table 2] Effectiveness of Exercise on Exercise Adherences and Health Self-Efficacy

\begin{tabular}{clllll}
\hline \multirow{2}{*}{ Variable } & \multicolumn{1}{c}{ Sub-Categories } & $\begin{array}{c}\text { Pre } \\
\mathrm{M}(\mathrm{SD})\end{array}$ & $\begin{array}{c}\text { Post } \\
\mathrm{M}(\mathrm{SD})\end{array}$ & $\mathrm{Z}$ & $\mathrm{P}$ \\
\hline \multirow{3}{*}{$\begin{array}{c}\text { Health } \\
\text { Self-Efficacy }\end{array}$} & Exercise & $2.92(.28)$ & $2.94(.35)$ & -.06 & .95 \\
& Psychological Well Being & $2.94(.29)$ & $3.02(.28)$ & -1.42 & .16 \\
& Nutrition & $2.78(.29)$ & $2.93(.31)$ & $-2.25^{*}$ & .03 \\
& Responsible Health Practices & $2.87(.35)$ & $2.95(.40)$ & -.95 & .34 \\
\hline \multirow{2}{*}{$\begin{array}{c}\text { Exercise } \\
\text { Adherences }\end{array}$} & Total Score & $2.88(.19)$ & $2.96(324)$ & -1.34 & .18 \\
& Exercise Abilities & $3.20(.67)$ & $3.24(.74)$ & -.41 & 0.69 \\
& Exercise Habits & $3.13(.68)$ & $3.43(.58)$ & $-.2 .63^{* *}$ & $<.01$ \\
& Exercise Environments & $3.49(.53)$ & $3.64(.50)$ & -.71 & .48 \\
& Exercise Partners & $2.92(.58)$ & $3.35(.67)$ & $-3.06^{* *}$ & $<.01$ \\
\hline${ }^{*} \mathrm{p}<.05,{ }^{* *} \mathrm{p}<.01$, & $3.38(.46)$ & $3.53(.58)$ & -1.48 & .14 \\
\hline & Total Score & $3.24(.42)$ & $3.44(.43)$ & $-2.75^{* *}$ & $<.01$ \\
\hline
\end{tabular}

\section{Discussion}

In this study, the effects of 12 weeks combined exercise on physical factors (weight, weight circumference, skeletal muscle mass, body fat mass) and psychological factors (health self-efficacy, willingness to exercise adherence) in middle-aged women.

The results showed that the middle-aged women who participated in combined exercise program experienced significant decrease in waist circumference and body fat mass and increase in skeletal muscle mass. They also showed significant decrease in TG and LDL-cholesterol. Consistent with the results of Kim[13] that the body fat ratio, WHR, and LDL cholesterol decreased significantly in middle-aged obesity women after participation in aerobic and combined exercise for 12 weeks. Lee et al.[24] investigated the metabolic syndrome factors in obesity women of each age group after participation in combined exercise composed of aerobic and resistance exercises for 12 weeks and reported decrease in body fat mass and WHR in 40's and 50's women and TG in 40's women, supported by results of this study.

Kim et al.[25] investigated the effect of eight weeks combined exercises composed of aerobic 
The Effects of Combined Exercise Program on the Body Composition, Metabolic Syndrome Risk Factor in Middleaged Women

and anaerobic exercises in postmenopausal women and reported significant decrease in TC, LDL-C, and TG and Joseph et al.[26] also reported significant decrease in TC, LDL-C, and TG in postmenopausal obesity women who participated in six months low-intensity exercise program. These results indicates that the combined exercise composed of two or more exercises is effective for postmenopausal women. In the health management of middle-aged women, the physical change of menopause is a very important factor. The middle aged women are highly likely to be exposed to diseases such as metabolic syndrome and cardiovascular disease[3] since the menopause accompanies physiological changes including decrease of vascular protection effect of estrogen hormone, change of blood lipid metabolism, visceral obesity. Combined exercise is effective in preventing metabolic syndrome through positive effects on basal metabolism, lipid metabolism, lipid metabolism, and muscle mass, and energy expenditure[27].

As evidenced by the results of this study, the combined exercise composed of aerobic and low-middle intensity exercises is effective in reducing triglycerides, an important predictors of metabolic syndrome, total cholesterol, low-density cholesterol, waist circumference, and body fat mass. The combined exercise program for middle-aged women significantly increased the health self-efficacy(nutrition), and the exercise adherence(exercise habit and exercise concern). Strategies using self-efficacy play an important role in the practice and continuity of the basic principles of obesity management, such as exercise and dietary control[28]. The health self-efficacy in this study had four sub-scales of exercise efficacy, psychological wellbeing efficacy, nutrition efficacy, and responsible health practice efficacy, among them, nutrition efficacy significantly increased after program. This is deemed to be due to the offering of diet information including nutritional consultation reflecting weight control demand of subjects and continuous offering of information through mobile apps. A study on 12-weeks nutritional education and exercise programs in middle-aged abdominal obesity women reported significant decrease in total cholesterol levels[29]. Considering the results of previous studies, it is interpreted that the offering of nutrition information had positive effect on combined exercise program. The combination of health management programs may have positive effects on body composition and blood lipid as well as health self-efficacy, thus strengthen the abilities to improve life habit and manage habit by themselves.

The exercise adherence, a consistent participation in exercise, is influenced by a variety of factors including age, occupation, residence exercise experience, and exercise environment[30]. Women, in particular, are less active than men, and were shown to give meaning to the motivation for exercise, presenting the 'no time' or 'annoyance' as the factors inhibiting exercise[30]. The levels of exercise habit and exercise concern, which are sub-scales of exercise 
adherence, significantly increased in this study. The results of this study support previous studies that middle-aged women with metabolic syndrome have a significant effect on exercise continuity[31][32]. The complex exercise program of this study strengthened the willingness to exercise adherence by providing the participants with a variety of exercises; therefore, it is suggested that allow them to exercise regularly and continuously and to select the appropriate exercise according to their own physical condition.

This exercise is more important in middle-aged women because they are subjected to various physical and psychological diseases due to menopause in the course of transition to elderly. Although the effects of exercise may be improved through manipulating the intensity, the number of times is more emphasized than the intensity, on which the willingness to participate in and interest of exercise had strong effects[23]. The combined exercise applied in this study resulted in the decrease an predictive factor of metabolic syndromes in middle-aged women, and improved the willingness of exercise adherence, however, failed in improving the health self-efficacy on exercise. It is urgent to provide a program, which contains measures such as behavior modification that induce adults to participate actively and self-directly in the exercise and lead healthy life by themselves. Future researches are recommended to investigate the effect of exercise widely by adding physical variables.

It is important to select exercises that reflect not only effects on body but also other various conditions such as form and environmental condition of exercise, willingness of exercise adherence. It is necessary, therefore to develop and apply proper exercise program by identifying the self-efficacy of the subject and various environmental factors so as to change the behavior in a desirable direction by maintaining regular physical activity[23].

The middle-age is a very important period to prepare for a successful elderly life. Considering that current middle-aged women are the subject of the aging society in the future, they should be able to manage their own health with correct awareness of health through regular exercise[33]. The development of the combined exercise program reflecting various factors for the health promotion of middle-aged women and the additional study, therefore, proposed.

\section{Conclusion}

This study examined the effects on physical (weight, weight circumference, skeletal muscle mass, body fat mass) and psychological (health self-efficacy, willingness to exercise adherence) factors after 12 weeks of complex exercise in middle-aged women. 


\section{The Effects of Combined Exercise Program on the Body Composition, Metabolic Syndrome Risk Factor in Middleaged Women}

The combined exercise program in middle-aged women decreased the level of triglyceride and cholesterol and waist circumference, which are risk factors of metabolic syndrome, and had positive effects on health self-efficacy and exercise adherence. The proposed program is expected to raise the willingness to participate in health management in middle-aged women who are experiencing many physical and psychological changes by providing positive effect on their health management and inducing continuous exercise adherence.

\section{Acknowledgement}

This study was funded by the research grants for new professors of Kyungnam University

\section{References}

[1] H. T. Kang, S. Y. Kim, J. Kim, J. Kim, J. Kim, H. A. Park, J. Shin, S. H. Cho, Y. Choi, J. Y. Shim, Clinical practice guideline of prevention and treatment for metabolic syndrome, Korean Journal of Family Practice, (2015), Vol.5, No.3, pp.375-420.

[2] A. R. Hong, S. Lim, Clinical characteristics of metabolic syndrome in Korea, and its comparison with other Asian countries, Journal of Diabetes Investigation, (2015), Vol.6, No.5, pp.508-515. DOI: 10.1111/jdi.12313

[3] M. S. Han, Metabolic Syndrome Emerging from Menopause, Journal of Menopausal Medicine, (2011), Vol.17, No.3. pp.127-135.

[4] S. R. Davis, C. Castelo-Branco, P. Chedraui, M. A. Lumsden, R. E. Nappi, D. Shah, P. Villaseca, Understanding weight gain at menopause, Climacteric, (2012), Vol.15, No.5, pp.419-448. DOI: $10.3109 / 13697137.2012 .707385$

[5] E. Yoon, J Lee, H Kang, E. Ahn, S. Woo, D. Kim, Optimal exercise volume for prevention, treatment of obesity and metabolic syndrome in central obese mid-Life women -Focused on pre-post-menopausal women-, The Korean Journal of Physical Education, (2008), Vol.47, No.6, pp.669-681. UCI: G704-000541.2008.47.6.023

[6] S. M. Grundy, H. B. Brewer, J. I. Cleeman, S. C. Smith, C. Lenfant, Definition of metabolic syndrome: report of the National Heart, Lung, and Blood Institute/American Heart Association conference on scientific issues related to definition, Circulation, (2004), Vol.109, No.3, pp.433-438. DOI: 10.1161/01.ATV.0000111245.75752.C6

[7] M. J. Kim, Midlife development, Kyomoonsa, (1989)

[8] P. Stiegler, A. Cunliffe, The role of diet and exercise for the maintenance of fat-free mass and resting metabolic rate during weight loss, Sports medicine, (2006), Vol.36, No.3, pp.239-262. DOI: 10.2165/00007256-200636030-00005

[9] S. Vaughan, M. Wallis, D. Polit, M. Steele, D. Shum, and N. Morris, The effects of multimodal exercise 
on cognitive and physical functioning and brain-derived neurotrophic factor in older women: a randomized controlled trial, Age and Ageing, (2014), Vol.43, pp.623-629, DOI: 10.1093/ageing/afu010

[10] D. J. Kim, The Relationship among Perceived Health Status, Life Satisfaction and Health Promotion Behavior in Physical Activity Participants in Middle Age, Journal of Sport and Leisure Studies, (2012), No.47, pp.503-518. UCI : G704-000763.2012..47.049

[11] Y. O. Joung, O. H. Sook, The Effects of Social support, Self-efficacy and Perceived health status on Aging anxiety of the Middle-aged women, Unpublished master's thesis, Journal of Korean Public Health Nursing (J Korean Pubilc Health Nurs), (2016), Vol.30, No.1, pp.30-41. UCI : G704-SER000009448.2016.30.1.010

[12] I. W. Kang, W. J. Cho, The Influence on Mental Health Status and Health-Related Quality of Life in Middle-Aged Women by The Regular Walking Exercise by Based on the Korea National Health and Nutrition Examination Survey(KNHANES V), Journal of Wellness (KSW), (2016), Vol.11, No.1, pp.207-215. UCI : G704-SER000002143.2016.11.1.012

[13] American College of Sports Medicine, ACSM's Guidelines for Exercise Testing and Prescription, 7th Edition, Lippincott Williams \& Wilkins, (2005)

[14] J. S. Kim, Effects of Exercise Type on Body Composition, Physical Fitness and Blood Variables in Obese Middle-aged Women, Journal of Sport and Leisure Studies, (2010), No..41, pp.963-973. DOI : 10.51979/KSSLS.2010.08.41.963

[15] D. Cho, M. Lee, E. Kim, G. Lee, A Comparative analysis on the Body Composition, Health-Related Fitness, and Social Physique Anxiety of Dance Singers after 12 Weeks Combined exercise, Journal of Korean Society for Rhythmic Exercises (JKSRE), (2011), Vol.4, No.1, pp.75-85.

[16] J. Pintar, R. Robertson, A. Kriska, E. Nagle, F. L. Goss, The influence of fitness and body weight on preferred exercise intensity, Medicine \& Science in Sports \& Exercise, (2006), Vol.38, No.5, pp.981-988. DOI: $10.1249 / 01 . m s s .0000218128 .66077 .97$

[17] S. K, Park, Y. Kwon, E. Kim, S. Lee, The Effect of Combined Training on Abdominal Fat, Physical Fitness and Resistin Concentration in Aged Visceral Obese Women, Korea Sport Research, (2006), Vol.17, No.3, pp.237-250. UCI : G704-001366.2006.17.3.031

[18] B. K. Yu, The Change of Severely Obese Middle Aged Women's Body Fat, Cardiovascular Strength and Blood Hormone on the Combined Exercise, Physical Activity and Nutrition (Phys Act Nutr), (2005), Vol.9, No.3, pp.253-259. UCI : G704-000668.2005.9.3.008

[19] Donna E. Shalala, Physical Activity and Health: A report of Surgeon General, Center for Disease Control and Prevention, (1999)

[20] J. M. Cholewa, F. E. Rossi, C. MacDonald, A. Hewins, S. Gallo, A. Micenski, B. I. Campbell, The Effects of Moderate- Versus High-Load Resistance Training on Muscle Growth, Body Composition, and Performance in Collegiate Women, Journal of Strength and Conditioning Research, (2018), Vol.32, No.6, pp.1511-1524. DOI: 10.1519/JSC.0000000000002048

[21] H. Becker, A. Stuifbergen, H. Oh, S. Hall, Self-Rated Abilities for Health Practices: A Health Self-Efficacy Measure, Health Values, (1993), Vol.17, No.5, pp.42-50.

[22] J. M. Choi, The Effects of college student' self-efficacy on their health promotion behavior, Ewha Womans University, Unpublished master's thesis, (2004) 
[23] S. H. Choi, (The) Development of a Korean exercise adherence scale, Seoul University, Unpublished Master's Thesis, (2005)

[24] S. Lee, M. Heo, S. Noh, The Effect on Body Composition and Metabolic Syndrome Factors of the 12-week combination exercise program by age Groups in Obese Women, The Korean Society of Sports Science, (2017), Vol.26, No.1, pp.1111-1122. DOI : 10.35159/kjss.2017.02.26.1.1111

[25] D. Kim, I. Baek, S. Seo, J. Kim, The Effects of Combined Exercise on Serum RBP4, Estradiol(E2) and HOMA-IR in Postmenopausal Women with Metabolic Syndrome and Abdominal Obesity, Journal of Physical Education and Sport \& Leisure Studies, (2014), Vol.21, No.1, pp.17-36.

[26] L. J. Joseph, R. L. Prigeon, J. B. Blumenthal, A. S. Ryan, A. P. Goldberg, Weight Loss and Low-Intensity Exercise for the Treatment of Metabolic Syndrome in Obese Postmenopausal Women, Journals of Gerontology Series A: Biological Sciences and Medical Sciences, (2011), Vol.66, No.9, pp.1022-1031. DOI: $10.1093 /$ gerona/glr093

[27] D. R. Dengel, J. M. Hagberg, P. J. Coon, D. T. Drinkwater, A. P. Goldberg, Effects of weight loss by diet alone or combined with aerobic exercise on body composition in older obese men, Metabolism: Clinical and Experimental, (1994), Vol.43, No.7, pp.867-871. DOI: 10.1016/0026-0495(94)90268-2

[28] J. A. James, Relations of changes in self-efficacy, exercise attendance, mood, and perceived and actual physical changes in obese women assessing treatment effect using tenets of self-efficacy theory, Journal of Social Behavioral and Health Sciences, (2007), Vol.1, No.1, pp.72-85.

[29] J. Kim, Y. Kweon, H. Jo, The Effect of 12 Weeks Nutrition Education and Combined Exercise on Serum Lipids and Appetite Hormone in the Middle Aged Obese Women, Journal of Sport and Leisure Studies, (2013), No.52, pp.815-825. DOI : 10.51979/KSSLS.2013.05.52.815

[30] Sports and Tourism, Survey on Citizen's Sports Participation, Ministry of Culture, (2012)

[31] M. Han, Relationship between physical self-efficacy and exercise adherence according to the self-management in the participant of life time sports, Dankook University, Master's Thesis, (2010)

[32] H. Yoo, (The) Effects of Sports Participation Related to Physical Self-efficacy and Exercise Adherences of Metabolic Syndrome Women, Kookmin University, Master's Thesis, (2016)

[33] R. Ma, Y. J. Jee, Effects of Combined Exercise on Exercise Adherences and Health Self-Efficacy in Middle-aged Women, International Journal of Advanced Nursing Education and Research, (2019), Vol.4, No.2, pp.45-50. DOI: 10.21742/ijaner.2019.4.1.08 